\title{
Constraints on a Vacuum Energy from Both SNIa and CMB Temperature Observations
}

\author{
Riou Nakamura, E. P. Berni Ann Thushari, Mikio Ikeda, and Masa-Aki Hashimoto \\ Department of Physics, Kyushu University, 6-10-1 Hakozaki, Higashi-ku, Fukuoka City 812-8581, Japan \\ Correspondence should be addressed to Riou Nakamura, riou@phys.kyushu-u.ac.jp
}

Received 28 February 2012; Accepted 1 May 2012

Academic Editor: Rob Ivison

Copyright ( $) 2012$ Riou Nakamura et al. This is an open access article distributed under the Creative Commons Attribution License, which permits unrestricted use, distribution, and reproduction in any medium, provided the original work is properly cited.

\begin{abstract}
We investigate the cosmic thermal evolution with a vacuum energy which decays into photon at the low redshift. We assume that the vacuum energy is a function of the scale factor that increases toward the early universe. We put on the constraints using recent observations of both type Ia supernovae (SNIa) by Union-2 compilation and the cosmic microwave background (CMB) temperature at the range of the redshift $0.01<z<3$. From SNIa, we find that the effects of a decaying vacuum energy on the cosmic expansion rate should be very small but could be possible for $z<1.5$. On the other hand, we obtain the severe constraints for parameters from the $\mathrm{CMB}$ temperature observations. Although the temperature can be still lower than the case of the standard cosmological model, it should only affect the thermal evolution at the early epoch.
\end{abstract}

\section{Introduction}

One of the biggest cosmological mysteries is the accelerating cosmic expansion which was discovered by the observations of distant type Ia supernovae (SNIa) starting from more than 10 years ago. For the origin of the accelerating expansion, the following possibilities have been proposed: the modified gravity, such as $f(R)$ gravity $[1,2]$, brane-world cosmology [3], inhomogeneous cosmology [4,5], and existence of unknown energy called as dark energy which is equivalent to a cosmic fluid with a negative pressure.

It is strongly suggested that the dark energy amounts to $70 \%$ of the total energy density of the universe from the astronomical observations such as SNIa [6-8], the anisotropy of cosmic microwave background (CMB) [9-11], and the baryon acoustic oscillation [12]. Although there are various theoretical models of dark energy (details are shown in a review $[13,14])$, its physical nature is still unknown. Considering above-referenced observations, we can not exclude the constant $\Lambda$ term which is the most simple model of dark energy.

The interacting dark energy with $\mathrm{CMB}$ photon has been discussed, whereas decaying vacuum into photon is related to a primordial light-element abundances [15] and the $\mathrm{CMB}$ intensity [16]. From the point of the thermodynamical evolution in the universe, a decaying vacuum energy modifies the temperature-redshift relation [17-19]. This modification affects the cosmic thermal evolution after a hydrogen recombination [20], such as formation of molecules [21, 22] and the first star [21]. In addition to this model, the angular power spectrum of $\mathrm{CMB}$ could be also modified [23]. In the previous analysis, thermal history at the higher redshift has been studied. However, as seen in [20,21], the time variation of temperature at low redshift differs from the model without a decaying vacuum.

In the present study, we update the observational consistency of a vacuum energy (hereafter we denote as $\Lambda$ ) coupled with $\mathrm{CMB}$ photon. Hereafter, we call this model D $\Lambda$ CDM (model). To examine the consistency with observations at low redshift, we focus on the cosmic evolution comparing both the type Ia supernovae and the redshift dependence of $\mathrm{CMB}$ temperatures. This is because the time dependence of the CMB temperature for a $\mathrm{D} \Lambda \mathrm{CDM}$ differs from CDM model with a constant $\Lambda$ term of which hereafter we call $\mathrm{S} \Lambda \mathrm{CDM}$ (model). In Section 2, formulation of D $\Lambda \mathrm{CDM}$ is reviewed. In Section 3, the $m-z$ relation is investigated for 
$\mathrm{D} \Lambda \mathrm{CDM}$ in a flat universe. Parameters inherent in this model are constrained from the CMB temperature observations in Section 4. Concluding remarks are given in Section 5.

\section{Dynamics of the Decaying $\Lambda$ Model}

The Einstein's field equation is written as follows (e.g., [24]):

$$
R_{\mu \nu}-\frac{1}{2} g_{\mu \nu} R=8 \pi G T_{\mu \nu}
$$

where $G$ is the gravitational constant and $T_{\mu \nu}$ is the energy momentum tensor. If we assume the perfect fluid, $T_{\mu \nu}$ is written as follows:

$$
T_{\mu \nu}=\operatorname{diag}(-\rho, p, p, p) .
$$

Here $\rho$ and $p$ are the energy density and the pressure, respectively. Note that we choose the unit of $c=1$.

The equation of motion is obtained with use of the Friedmann-Robertson-Walker metric of the homogeneous and isotropic principle:

$$
d s^{2}=-d t^{2}+a(t)^{2}\left[\frac{d r^{2}}{1-k r^{2}}+r^{2} d \theta^{2}+r^{2} \sin ^{2} \theta d \phi^{2}\right],
$$

where $a(t)$ is the scale factor and $k$ is the specific curvature constant. This leads to the Friedmann equations:

$$
H^{2} \equiv\left(\frac{\dot{a}}{a}\right)^{2}=\frac{8 \pi G}{3} \rho-\frac{k}{a^{2}} .
$$

From the conservation's law of energy, we can obtain the equation of the energy density:

$$
\dot{\rho}=-3(1+w) \frac{\dot{a}}{a} \rho,
$$

where $w$ is the coefficients of the equation of state, $p=w \rho$, and defined by

$$
w \equiv \begin{cases}\frac{1}{3} & \text { for photon and neutrino } \\ 0 & \text { for baryon and cold dark matter } \\ -1 & \text { for vacuum energy }\end{cases}
$$

We take the component of the energy density as

$$
\rho=\rho_{\gamma}+\rho_{\nu}+\rho_{m}+\rho_{\Lambda}
$$

where $\gamma, v, m$, and $\Lambda$ indicate photon, neutrino, nonrelativistic matter (baryon plus cold dark matter), and the vacuum, respectively. Here we neglect the energy contribution of $\Lambda$ on other components except for photon: the $\rho_{\nu}$ and $\rho_{m}$ evolves as $\rho_{\nu} \propto a^{-4}$ and $\rho_{m} \propto a^{-3}$, respectively. From (5), the equation of the photon coupled with $\Lambda$ is written as

$$
\dot{\rho}_{\gamma}+4 H \rho_{\gamma}=-\dot{\rho}_{\Lambda}
$$

Let us define the parameter of the energy density as follows:

$$
\Omega_{i} \equiv \frac{\rho_{i}}{\rho_{c r}}=\frac{8 \pi G}{3 H_{0}^{2}} \rho_{i}
$$

where $\rho_{c r}$ is the critical density defined by the present Hubble constant $H_{0}$. We can rewrite (8) as follows (for details also see $[22,23])$ :

$$
\frac{d \Omega_{\gamma}}{d a}+4 \frac{\Omega_{\gamma}}{a}=-\frac{d \Omega_{\Lambda}}{d a} .
$$

Models with time-dependent $\Lambda$-term have been studied as summarized in $[25,26]$. We adopt the energy of the vacuum which varies with the scale factor [20-23]:

$$
\Omega_{\Lambda}(a)=\Omega_{\Lambda 1}+\Omega_{\Lambda 2} a^{-m}
$$

where $\Omega_{\Lambda 1}, \Omega_{\Lambda 2}$, and $m$ are constants. Note that the present value of $\Omega_{\Lambda}$ is expressed by $\Omega_{\Lambda 0}=\Omega_{\Lambda 1}+\Omega_{\Lambda 2}$.

Integrating (10) with (11), we obtain the photon energy density as a function of $a[22]$ :

$$
\Omega_{\gamma}= \begin{cases}{\left[\Omega_{\gamma 0}+\alpha\left(a^{4-m}-1\right)\right] a^{-4}} & (m \neq 4), \\ \left(\Omega_{\gamma 0}+4 \Omega_{\Lambda 2} \ln a\right) a^{-4} & (m=4),\end{cases}
$$

where $\Omega_{\gamma 0}=2.471 \times 10^{-5} h^{-2}\left(T_{\gamma 0} / 2.725 \mathrm{~K}\right)^{4}$ is the present photon energy density, $h$ is the normalized Hubble constant ( $\left.H_{0}=100 \mathrm{~h} \mathrm{~km} / \mathrm{sec} / \mathrm{Mpc}\right)$, and $T_{\gamma 0}$ is the CMB temperature at the present epoch. We define $\alpha$ as $\alpha=m \Omega_{\Lambda 2} /(4-m)$.

Since we assume the flat geometry $(k=0)$ in this work, we can write the condition of $\Omega$ s as follows:

$$
\Omega_{m 0}+\Omega_{\Lambda 1}+\Omega_{\Lambda 2}=1 .
$$

From now on, we adopt the present cosmological parameters: Hubble constant $h=0.738$ [27, 28], and the present density parameter of matter $\Omega_{m}=0.2735$ [11]. The present temperature is $T_{\gamma 0}=2.725 \mathrm{~K}$ observed by COBE [29]. In our study, we search the parameter regions in the range of $\Omega_{\Lambda 2}$ and $m: 10^{-7}<\Omega_{\Lambda 2}<10^{-2}$ and $0<m<4$. The range of $m$ has already been obtained from the previous analysis [23].

Here we note the range of $\Omega_{\Lambda 2}$ in the present analysis. Kimura et al. [20] found that the radiation temperature (i.e., the energy density of radiation) in D $\Lambda$ CDM model becomes numerically negative at a point of $a<1$ when $m$ and/or $\Omega_{\Lambda 2}$ is too large. To avoid this unreasonable situation, that is, $T_{\gamma}<$ 0 , Nakamura et al. [23] imposed the limit of $\Omega_{\Lambda 2}$ and $m$ as follows:

$$
\begin{gathered}
\alpha<\Omega_{\gamma 0} \quad(m<4), \\
\Omega_{\Lambda 2}<\Omega_{\gamma 0} / 92 \quad(m=4) .
\end{gathered}
$$

From the condition (14), we obtain the limit of $\Omega_{\Lambda 2}$ as $4.9 \times$ $10^{-7}$ for $m=4$. We can consider that the effect of $\Omega_{\Lambda 2}$ is negligible when $\Omega_{\Lambda 2}$ is smaller than $10^{-7}$.

\section{SNIa Constraints}

The cosmic distance measures depend sensitively on the spatial curvature and expansion dynamics of the models. Therefore, the magnitude-redshift relation for distant standard candles are proposed to constrain the cosmological parameters. 


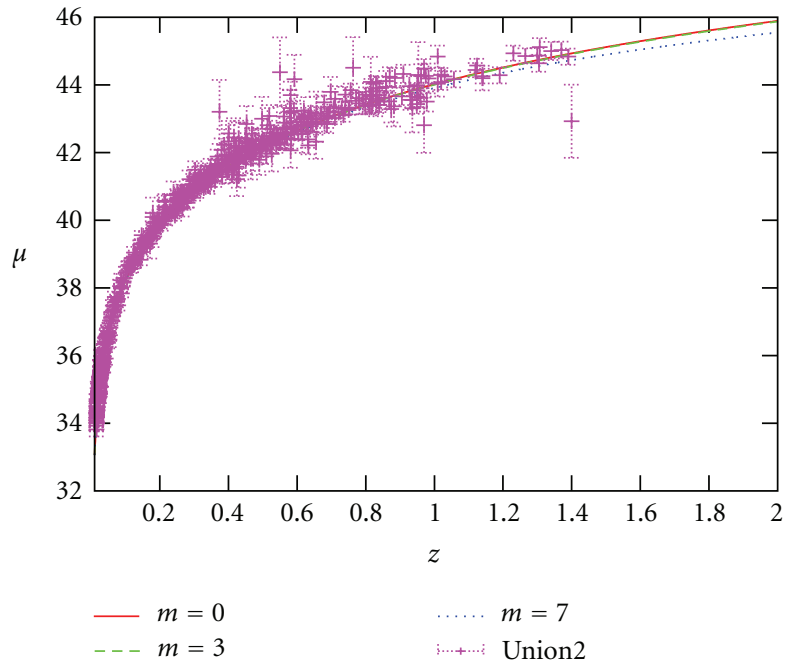

FIGURE 1: Illustration of the magnitude-redshift relation in S $\Lambda$ CDM model $(m=0)$ and $\mathrm{D} \Lambda \mathrm{CDM}$ with $\Omega_{\Lambda 2}=10^{-2}$ compared with SNIa observations (cross-shaped ones with error bars). Note that the theoretical curve for $m=3$ has no difference with that of $m=0$.

To calculate the effects of the comic expansion at low- $z$, we calculate the theoretical distance modules,

$$
\mu_{\mathrm{th}}=m_{B}-M=5 \log d_{L}+25,
$$

where $m_{B}, M$, and $d_{L}$ are the apparent and absolute magnitudes, and the luminosity distance, respectively. Here $d_{L}$ is related to the radial distance $r$ in the metric as follows [24]:

$$
d_{L}=(1+z) r .
$$

We can obtain $r$ as follows:

$$
r=\int \frac{d t}{a(t)}=\frac{1}{H_{0}} \int \frac{d z}{E(z)},
$$

where $z$ is the redshift defined by $a=1 /(1+z)$. Now $E(z)$ is defined as

$$
E(z)^{2}=\left(\frac{H(z)}{H_{0}}\right)^{2}=\Omega_{m 0}(1+z)^{3}+\Omega_{\Lambda 1}+\Omega_{\Lambda 2}(1+z)^{m} .
$$

Then, (16) is rewritten as follows:

$$
d_{L}=\frac{(1+z)}{H_{0}} \int \frac{d z}{E(z)} .
$$

As a consequence, the theoretical distance modules are calculated by (15) and (19).

Recently, the Supernovae Cosmology Project (SCP) collaboration released their Union2 sample of 557 SNIa data [8]. The Union2 compilation is the largest published and spectroscopically confirmed SNIa sample. The observations are in the redshift range of $0.01<z<2$. For the SNIa data set, we evaluate $\chi^{2}$ value of the distance modules,

$$
\chi^{2}=\sum_{i=1}^{N} \frac{\left(\mu_{\mathrm{th}, i}-\mu_{\mathrm{obs}, i}\right)^{2}}{\sigma_{\mu_{\mathrm{obs}, i}}^{2}+\sigma_{v, i}^{2}},
$$

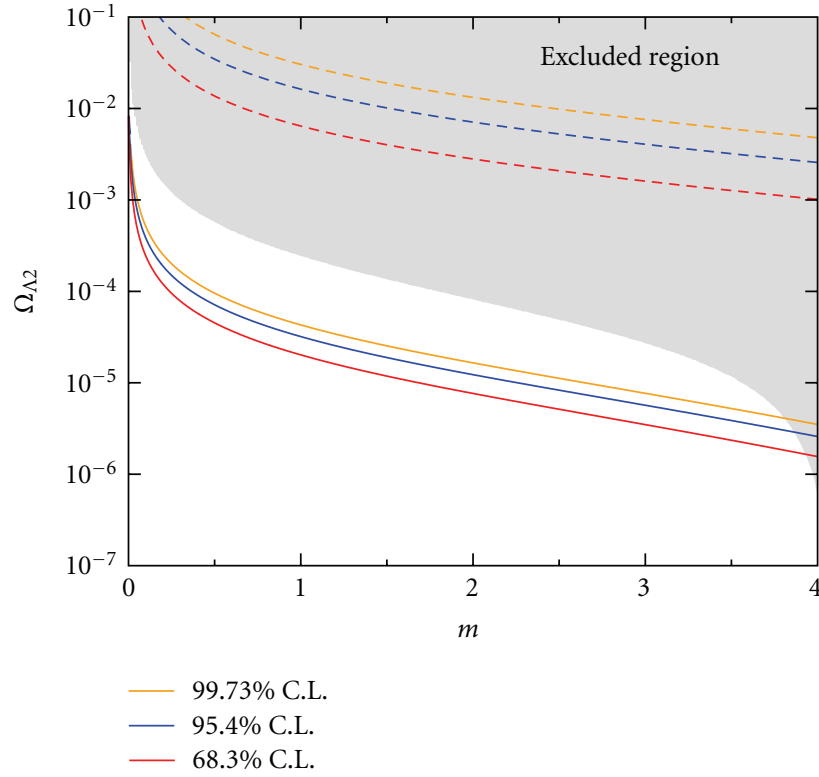

Figure 2: Constraints on the $m-\Omega_{\Lambda 2}$ plane from the recent observations of $\mathrm{CMB}$. The lines indicate upper bounds of 1,2 , and $3 \sigma$ confidence level. The dashed and solid lines are the limits deduced from SNIa and CMB temperature observations. The shaded region shows the excluded region obtained from [23].

where $\mu_{\mathrm{obs}, i}$ and $\mu_{\mathrm{th}, i}$ are the observed and the theoretical values of the distance modules. $\sigma_{v}$ is the dispersion in the redshift due to the peculiar velocity $v$ is written as:

$$
\sigma_{v}=\left(\frac{v}{c} \frac{d \mu_{\mathrm{th}}}{d z}\right)
$$

We adopt $v=300 \mathrm{~km} \mathrm{sec}^{-1}$ [40]. The total number of the united sample $N$ is 557 for the present analysis.

We confirm that $\Omega_{\Lambda 2}$ has done some contribution to change the $\mu-z$ relation. When $\Omega_{\Lambda 2}$ increases, the expansion rate in the universe decreases, which is similar to the behavior of the matter dominant universe in the Friedmann model.

Figure 1 indicates the relation between the distance modules and the redshift against the SNIa observations in terms of $\mathrm{S} \Lambda \mathrm{CDM}$ and $\mathrm{D} \Lambda \mathrm{CDM}$ with several values of $m$ for a fixed value of $\Omega_{\Lambda_{2}}=10^{-2}$. As the value of $m$ increases, $\mu$ tends to decrease because of the increasing cosmic expansion rate. We recognize that $m$ is not seriously effective to change the $\mu-z$ relation. Even if we chose the larger value of $m>4$, the effect is still small.

Figure 2 shows the allowed parameter region due to the $\chi^{2}$ fitting of $(20)$ in D $\Lambda$ CDM from SNIa constraints. We obtain the minimum value of $\chi^{2}=677$ (the reduced $\chi_{r}^{2}$ is defined to be $\chi_{r}^{2}=\chi^{2} / N \simeq 1.215$, where $N$ is the degree of freedom). The best fit parameter region of $\Omega_{\Lambda 2}$ for $m<4$ is investigated as $\Omega_{\Lambda 2}<4.5 \times 10^{-3}$ at $1 \sigma$ confidence level (C.L.). Since the parameter region is very loose compared with the previous analysis $[22,23]$, this result implies that the decaying- $\Lambda$ has minor effects on the expansion rate at $z<2$. 


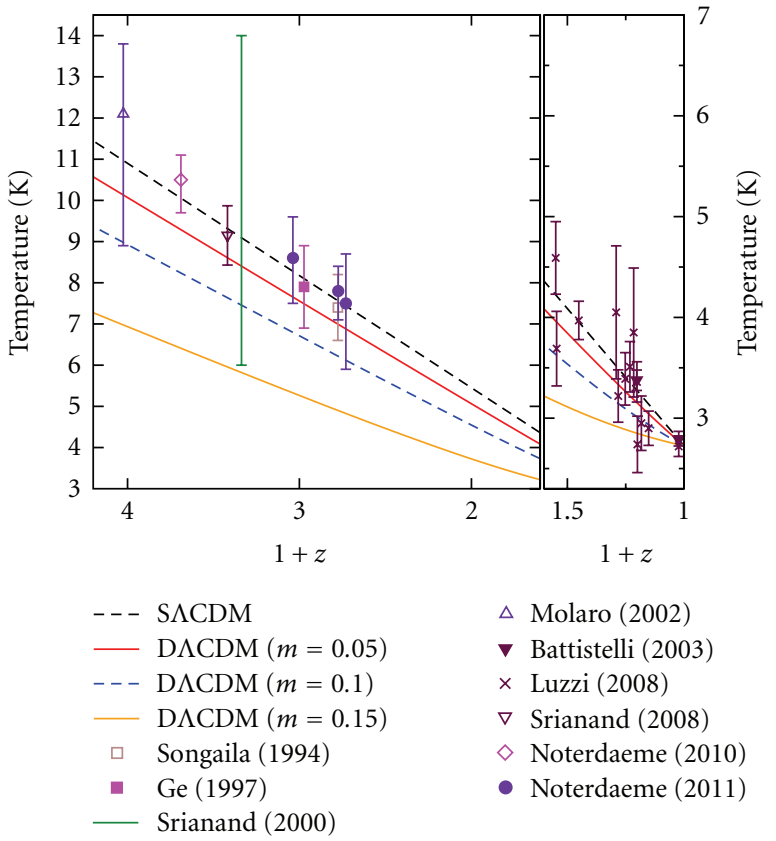

FIGURE 3: Illustration of the temperature evolutions compared with CMB observations. The left panel is the results at $z>0.6$. The right is same, but at $z<0.6$.

\section{Temperature Constraints}

It has been shown that the decaying- $\Lambda$ term affects the photon temperature evolution at the early epoch [20]. In this section, we study about the consistency of $\mathrm{D} \Lambda \mathrm{CDM}$ with recent temperature observations.

The temperature evolution is obtained from (10). Following the Stefan-Boltzmann Law, $\rho_{\gamma} \propto T_{\gamma}^{4}$, the relation between the photon temperature and the cosmological redshift is obtained as follows [22, 23]:

$$
\begin{gathered}
T_{\gamma}(z)=T_{\gamma 0}(1+z)\left(1+\frac{\Omega_{\gamma \Lambda}}{\Omega_{\gamma 0}}\right)^{1 / 4}, \\
\Omega_{\gamma \Lambda} \equiv \begin{cases}\alpha\left((1+z)^{m-4}-1\right) & (m \neq 4), \\
-4 \Omega_{\Lambda 2} \ln (1+z) & (m=4) .\end{cases}
\end{gathered}
$$

From (22), the temperature-redshift relation at higher- $z$ approaches that of $S \Lambda \mathrm{CDM}$ model, $T_{\gamma} \propto(1+z)$. At lower- $z$, the temperature evolution deviates from the proportional relation.

In the meanwhile, recently, the temperatures at higher- $z$ are observed using the molecules such as the fine structure of excitation levels of the neutral or ionized carbon [30-34] and the rotational excitation of CO [35-37] and SunyaevZel'dovich (S-Z) effect $[38,39]$. Furthermore, there are a lot of temperature observations for the lower- $z$ owing to the S-Z effect as shown in Table 1. Therefore, we expect to constrain more severely the allowable region on the $m-\Omega_{\Lambda 2}$ plane from the new temperature observations.

Figure 3 illustrates the temperature evolution in $\mathrm{S} \Lambda \mathrm{CDM}$ and $\mathrm{D} \Lambda \mathrm{CDM}$ model with $\Omega_{\Lambda 2}=10^{-2}$. It is shown that the
TABLE 1: Observational temperatures obtained from molecular excitation levels and S-Z effects.

\begin{tabular}{lcc}
\hline$z_{\text {obs }}$ & $T[\mathrm{~K}]$ & Reference \\
\hline 1.776 & $7.4 \pm 0.8$ & $\mathrm{CI}[30]$ \\
1.9731 & $7.9 \pm 1.0$ & $\mathrm{CI}[31]$ \\
2.3371 & $6.0<T[\mathrm{~K}]<14.0$ & $\mathrm{CI}[32]$ \\
3.025 & $12.1_{-3.2}^{+1.7}$ & $\mathrm{C}+[33]$ \\
1.77654 & $7.2 \pm 0.8$ & $\mathrm{CI}[34]$ \\
2.4184 & $9.15 \pm 0.72$ & $\mathrm{CO}[35]$ \\
2.6896 & $10.5_{-0.6}^{+0.8}$ & $\mathrm{CO}[36]$ \\
\hline 1.7293 & $7.5_{-1.6}^{+1.2}$ & $\mathrm{CO}[37]$ \\
1.7738 & $7.8_{-0.7}^{+0.6}$ & \\
2.0377 & $8.6_{-1.1}^{+1.0}$ & $\mathrm{~S}-\mathrm{Z}[38]$ \\
\hline 0.203 & $3.377_{-0.102}^{+0.101}$ & \\
0.0231 & $2.789_{-0.065}^{+0.080}$ & \\
\hline 0.023 & $2.72 \pm 0.10$ & \\
0.152 & $2.90 \pm 0.17$ & \\
0.183 & $2.95 \pm 0.27$ & \\
0.20 & $2.74 \pm 0.28$ & \\
0.202 & $3.36 \pm 0.2$ & \\
0.216 & $3.85 \pm 0.64$ & \\
0.232 & $3.51 \pm 0.25$ & \\
0.252 & $3.39 \pm 0.26$ & \\
0.282 & $3.22 \pm 0.26$ & \\
0.291 & $4.05 \pm 0.66$ & \\
0.451 & $3.97 \pm 0.19$ & \\
0.546 & $3.69 \pm 0.37$ & \\
0.55 & $4.59 \pm 0.36$ & \\
\hline & & \\
\hline & & \\
\hline & & \\
\hline & &
\end{tabular}

larger value of $m$ results in the lower- $T_{\gamma}$. When we adopt $m=0.15$, the theoretical curve of $T_{\gamma}$ should be inconsistent with observations even for the temperature of the largest uncertainty [32]. We can conclude that the deviation from $\mathrm{S} \Lambda \mathrm{CDM}$ model might be small for $m<0.1$.

Using the recent observations of the CMB temperature, whose accuracy has been improved quantitatively, we can put severe constraints on the temperature evolution in $\mathrm{D} \Lambda \mathrm{CDM}$ model. We calculate $\chi^{2}$-analysis as follows:

$$
\chi^{2}=\sum_{i}^{N} \frac{\left(T_{\gamma}(z)-T_{\mathrm{obs}, i}\right)^{2}}{\sigma_{i}^{2}},
$$

where $T_{\gamma}(z)$ is theoretical temperature calculated by (22) and $T_{\mathrm{obs}, i}$ the observational data as shown in Table 1. $\sigma_{i}$ is the uncertainty of the observation. $N=25$ is the number of the observational data. Note that the result of Srianand et al. [32] does not have best-fit value. Therefore, we assume the best-fit value is the same as the mean value, $T_{\text {Srianand }}=10.0 \pm 4.0 \mathrm{~K}$.

In Figure 2, we show the limits in the $m-\Omega_{\Lambda 2}$ plane calculated by (23). The shaded region indicates the parameter regions which should be excluded and are from (14). We can obtain the allowed parameter range as follows:

$$
\Omega_{\Lambda 2}<6.1 \times 10^{-4} \text { at } 1 \sigma \text { C.L. }, \quad 1.7 \times 10^{-3} \text { at } 2 \sigma \text { C.L. }
$$


Although temperature constraints give the upper bound, we can obtain the parameter range which is more severe than that obtained in the previous temperature constraint [22].

Finally, we identify the influence of temperature observations at $z<0.6$ by S-Z effect. We also calculate the $\chi^{2}$-fitting (23) using observations at $z>0.6$ and $z<0.6$, separately. As the results, we obtain the upper bound of $1 \sigma(2 \sigma)$ C.L. as follows:

$$
\begin{gathered}
\Omega_{\Lambda 2}<2.8 \times 10^{-3}\left(4.7 \times 10^{-3}\right) \quad \text { by S }-\mathrm{Z} \text { at } z<0.6, \\
\Omega_{\Lambda 2}<5.5 \times 10^{-4}\left(1.9 \times 10^{-3}\right) \quad \text { by CI, CO at } z>0.6 .
\end{gathered}
$$

These results suggested that the temperature observations at a higher- $z$ are important to constrain $\mathrm{D} \Lambda \mathrm{CDM}$ model.

\section{Concluding Remarks}

We have investigated the consistency of the decaying- $\Lambda$ model with both the $\mu-z$ relation of SNIa and CMB temperature. We obtain the upper bound of our model parameters from both observations. First, we have constrained our model from SNIa: we can obtain the upper bound of parameters, which region is wider compared with previous constraints $[22,23]$. From this result, a decaying- $\Lambda$ has minor effects on the cosmic expansion at low- $z$ and its parameters cannot be well constrained.

On the other hand, we can obtain more severe parameter constraints from CMB temperature. We acquire the limit of $\Omega_{\Lambda 2}$ as $\Omega_{\Lambda 2}<1.7 \times 10^{-3}$ at $2 \sigma$ C.L. From this result, the temperature can become lower by a few percentages. That should affect the hydrogen recombination. We note that we cannot get the bound of $m$. Because change in $\Omega_{\Lambda 2}$ cancels the effects of the variation of $m$.

Our parameter is mainly obtained by the observations at $z>2$. It has been suggested that the observations of the temperature at higher redshift is important to constrain the cosmological models with energy flow into photon [23]. The upper limit of $\Omega_{\Lambda 2}$ obtained from the present constraints is still larger than the previous constraints of $\Omega_{\Lambda 2}<1.7 \times 10^{-4}$ at $95.4 \%$ C.L. [23]. The analysis using the CMB temperature fluctuation of the recent observation seems to be unable to give the tight constraints. We plan to perform more detailed analysis, for instance using $\mathrm{CMB}$ anisotropy of the newest WMAP data $[10,11]$.

\section{Acknowledgment}

This work has been supported in part by a Grant-in-Aid for Scientific Research (18540279, 19104006, and 21540272) of the Ministry of Education, Culture, Sports, Science and Technology of Japan.

\section{References}

[1] A. de Felice and S. Tsujikawa, " $f(R)$ theories," Living Reviews in Relativity, vol. 13, no. 3, pp. 1-161, 2010.

[2] T. P. Satiriou and V. Faraoni, " $f(R)$ theories of gravity," Reviews of Modern Physics, vol. 82, pp. 451-497, 2010.
[3] K. Umezu, K. Ichiki, T. Kajino, G. J. Mathews, R. Nakamura, and M. Yahiro, "Observational constraints on accelerating brane cosmology with exchange between the bulk and brane," Physical Review D, vol. 73, no. 6, Article ID 063527, 2006.

[4] H. Alnes, M. Amarzguioui, and O. Gron, "An inhomogeneous alternative to dark energy?” Physical Review D, vol. 73, Article ID 083519, 2006.

[5] A. E. Romano, "Lemaitre-Tolman-Bondi universes as alternatives to dark energy: does positive averaged acceleration imply positive cosmic acceleration?" Physical Review D, vol. 75, no. 4, Article ID 043509, 2007.

[6] S. Perlmutter, G. Aldering, G. Goldhaber et al., "Measurements of $\Omega$ and $\Lambda$ from 42 high-redshift Supernovae," Astrophysical Journal, vol. 517, no. 2, pp. 565-586, 1999.

[7] A. G. Riess, A. V. Filippenko, P. Challis et al., "Observational evidence from supernovae for an accelerating universe and a cosmological constant," Astronomical Journal, vol. 116, no. 3, pp. 1009-1038, 1998.

[8] R. Amanullah et al., "Spectra and light curves of six type Ia Supernovae at $0.511<\mathrm{z}<1.12$ and the Union2 compilation," The Astrophysical Journal, vol. 716, no. 1, article 712, 2010.

[9] M. L. Brown, P. Ade, J. Bock et al., "Improved measurements of the temperature and polarization of the cosmic microwave background from quad," Astrophysical Journal, vol. 705, no. 1, pp. 978-999, 2009.

[10] N. Jarosik, C. L. Bennett, J. Dunkley et al., "Seven-year Wilkinson microwave anisotropy probe (WMAP) observations: sky maps, systematic errors, and basic results," Astrophysical Journal Suppl., vol. 192, 15 pages, 2011.

[11] E. Komatsu, K. M. Smith, J. Dunkley et al., "Even-year Wilkinson microwave anisotropy probe (WMAP) observations: cosmological interpretation," Astrophysical Journal Suppl., vol. 192, p. 18, 2011.

[12] W. J. Percival, B. A. Reid, D. J. Eisenstein et al., "Baryon acoustic oscillations in the Sloan Digital Sky Survey Data Release 7 galaxy sample," Monthly Notices of the Royal Astronomical Society, vol. 401, no. 4, pp. 2148-2168, 2010.

[13] P. J. E. Peebles and B. Ratra, "The cosmological constant and dark energy," Reviews of Modern Physics, vol. 75, pp. 559-606, 2003.

[14] E. J. Copeland, M. Sami, and S. Tsujikawa, "Dynamics of dark energy," International Journal of Modern Physics D, vol. 15, no. 11, pp. 1753-1935, 2006.

[15] K. Freese, F. C. Adams, J. A. Frieman, and E. Mottola, "Cosmology with decaying vacuum energy," Nuclear Physics, B, vol. 287, pp. 797-814, 1987.

[16] J. M. Overduin, P. S. Wesson, and S. Bowyer, "Constraints on vacuum decay from the microwave background," Astrophysical Journal, vol. 404, no. 1, pp. 1-7, 1993.

[17] J. A. S. Lima, "Thermodynamics of decaying vacuum cosmologies," Physical Review D, vol. 54, no. 4, pp. 2571-2577, 1996.

[18] P. Jetzer, D. Puy, M. Signore, and C. Tortora, "Limits on decaying dark energy density models from the CMB temperatureredshift relation," General Relativity and Gravitation, vol. 43, no. 4, pp. 1083-1093, 2011.

[19] P. Jetzer and C. Tortora, "Constraints from the CMB temperature and other common observational data sets on variable dark energy density models," Physical Review D, vol. 84, Article ID 043517, 2011.

[20] K. Kimura, M. Hashimoto, K. Sakoda, and K. Arai, "Effects on the temperatures of a variable cosmological term after recombination," Astrophysical Journal Letters, vol. 561, no. 1, pp. L19-L22, 2001. 
[21] M. Hashimoto, T. Kamikawa, and K. Arai, "Effects of a decaying cosmological term on the formation of molecules and first objects," Astrophysical Journal, vol. 598, no. 1, pp. 13-19, 2003.

[22] D. Puy, "Thermal balance in decaying $\Lambda$ cosmologies," Astronomy and Astrophysics, vol. 422, pp. 1-9, 2004.

[23] R. Nakamura, M.-A. Hashimoto, and K. Ichiki, "Cosmic microwave background constraints on a decaying cosmological term related to the thermal evolution," Physical Review D, vol. 77, no. 12, Article ID 123511, 2008.

[24] S. Weinberg, Cosmology, Oxford University Press, Oxford, UK, 2008.

[25] J. M. Overduin and F. I. Cooperstock, "Evolution of the scale factor with a variable cosmological term," Physical Review D, vol. 58, no. 4, Article ID 043506, 3 pages, 1998.

[26] V. Sahni and A. Starobinsky, "The case for a postive cosmological $\lambda$-term," International Journal of Modern Physics D, vol. 9, pp. 373-443, 2000.

[27] A. G. Riess et al., "A 3\% solution: determination of the Hubble constant with the Hubble space telescope and wide field camera 3," The Astrophysical Journal, vol. 730, p. 119, 2011.

[28] A. G. Riess et al., "Erratumml: A 3\% solution: determination of the Hubble constant with the Hubble space telescope and wide field camera 3," The Astrophysical Journal, vol. 732, p. 129, 2011.

[29] J. C. Mather, D. J. Fixsen, R. A. Shafer, C. Mosier, and D. T. Wilkinson, "Calibrator design for the COBE Far Infrared Absolute Spectrophotometer (FIRAS)," Astrophysical Journal, vol. 512, no. 2, pp. 511-520, 1999.

[30] A. Songaila, L. L. Cowie, S. Vogt et al., "Measurement of the microwave background temperature at a redshift of 1.776," Nature, vol. 371, no. 6492, pp. 43-45, 1994.

[31] J. Ge, J. Bechtold, and J. H. Black, "A new measurement of the cosmic microwave background radiation temperature at $z=$ 1.97," Astrophysical Journal, vol. 474, no. 1, pp. 67-73, 1997.

[32] R. Srianand, P. Petitjean, and C. Ledoux, "The cosmic microwave background radiation temperature at a redshift of 2.34," Nature, vol. 408, no. 6815, pp. 931-935, 2000.

[33] P. Molaro, S. A. Levshakov, M. Dessauges-Zavadsky, and S. D'Odorico, "The cosmic microwave background radiation temperature at zabs = 3.025 toward QSO 0347-3819," Astronomy and Astrophysics, vol. 381, no. 3, pp. L64-L67, 2002.

[34] J. Cui, J. Bechtold, G. E. Jian, and D. M. Meyer, "Molecular hydrogen in the damped Ly $\alpha$ absorber of Q1331+170," Astrophysical Journal, vol. 633, no. 2, pp. 649-663, 2005.

[35] R. Srianand, P. Noterdaeme, C. Ledoux, and P. Petitjean, "First detection of $\mathrm{CO}$ in a high-redshift damped Lyman- $\alpha$ system," Astronomy and Astrophysics, vol. 482, no. 3, pp. L39-L42, 2008.

[36] P. Noterdaeme, P. Petitjean, C. Ledoux, S. López, R. Srianand, and S. D. Vergani, "A translucent interstellar cloud at $z=2.69$ $\mathrm{CO}, \mathrm{H}_{2}$, and HD in the line-of-sight to SDSS J123714.60+ 064759.5," Astronomy and Astrophysics, vol. 523, article A80, 2010.

[37] P. Noterdaeme, P. Petitjean, R. Srianand, C. Ledoux, and S. López, "The evolution of the cosmic microwave background temperature: measurements of TCMB at high redshift from carbon monoxide excitation," Astronomy and Astrophysics, vol. 526, no. 11, article L7, 2011.

[38] E. S. Battistelli, M. De Petris, L. Lamagna et al., "Cosmic microwave background temperature at galaxy clusters," Astrophysical Journal Letters, vol. 580, no. 2 II, pp. L101-L104, 2002.

[39] G. Luzzi, M. Shimon, L. Lamagna et al., "Redshift dependence of the cosmic microwave background temperature from sunyaev-zeldovich measurements," Astrophysical Journal, vol. 705, no. 2, pp. 1122-1128, 2009.
[40] R. Kessler, A. C. Becker, D. Cinabro et al., "First-year sloan digital sky survey-ii supernova results: Hubble diagram and cosmological parameters," Astrophysical Journal Suppl., vol. 185, no. 1, pp. 32-84, 2009. 

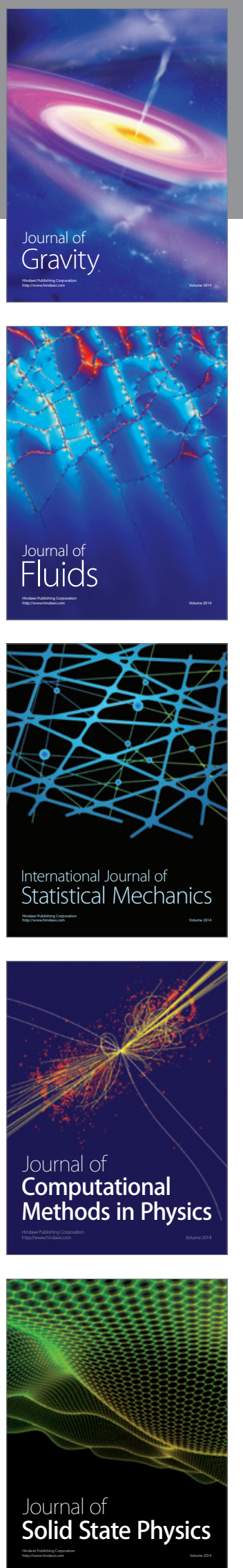

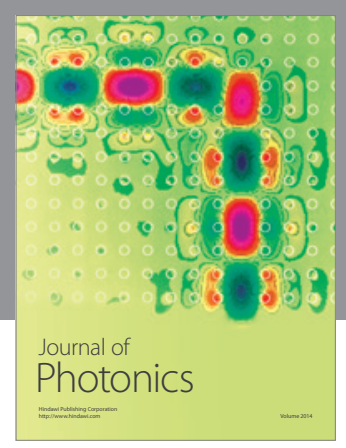

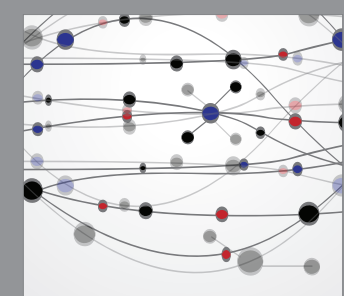

The Scientific World Journal
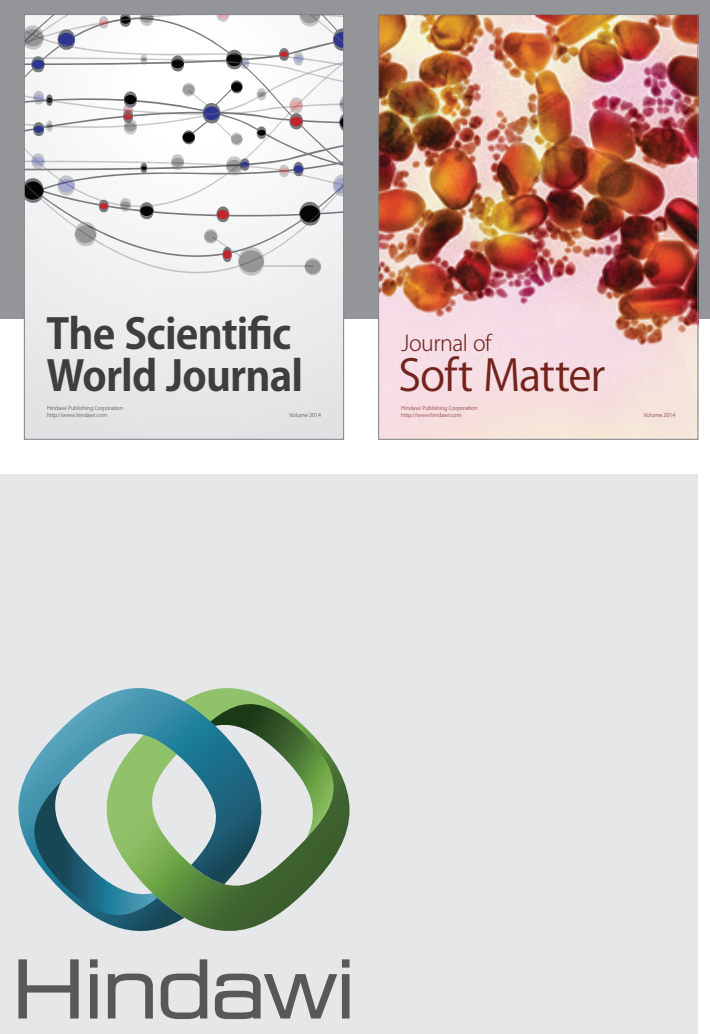

Submit your manuscripts at

http://www.hindawi.com
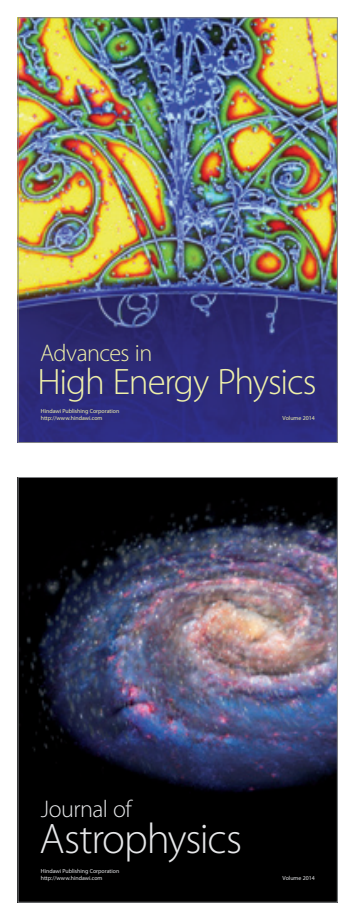
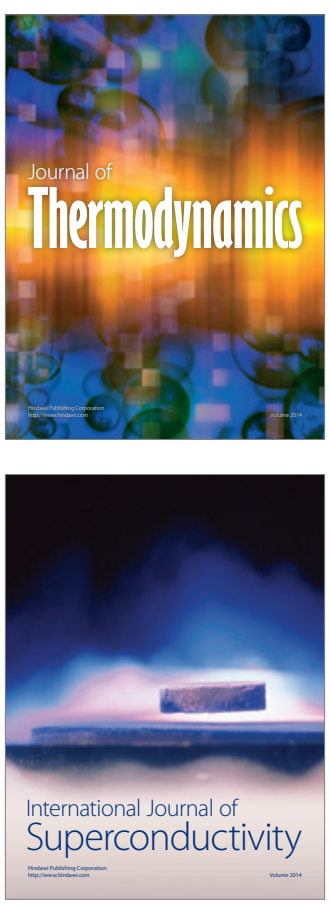
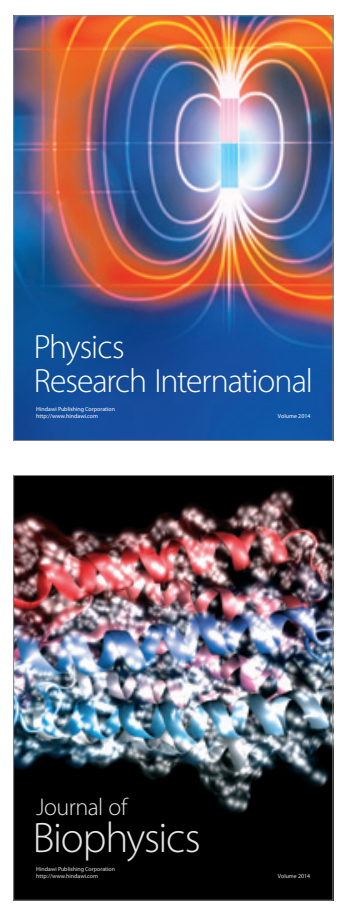
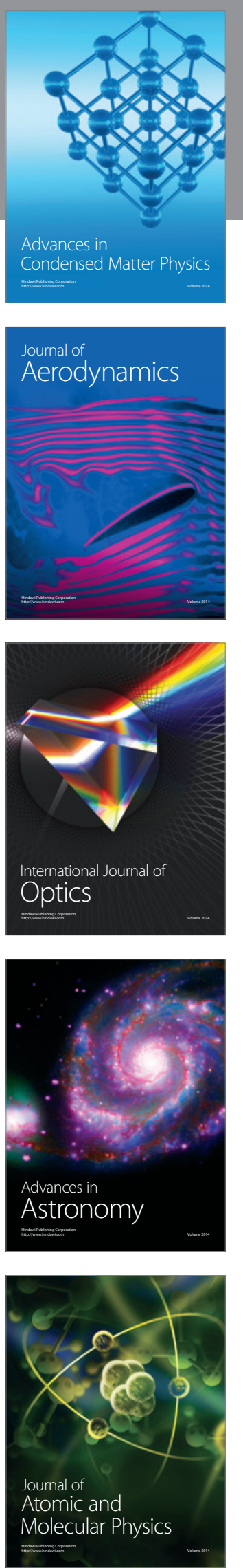